\title{
SM and Higgs at the HL-LHC
}

\author{
Tae Jeong Kim on behalf of the ATLAS and CMS collaborations* \\ Hanyang University \\ E-mail: taekim@hanyang.ac.kr
}

\begin{abstract}
After the Higgs discovery, more data was required not only to check the consistency with the Standard Model (SM) but also to search for possible new physics. At the High Luminosity LHC (HL-LHC), the LHC will reach a peak instantaneous luminosity of $7.5 \times 10^{34} \mathrm{~cm}^{-2} \mathrm{~s}^{-1}$ in the middle of 2026. This upgrade will lead us to data corresponding an integrated luminosity of 3000 $\mathrm{fb}^{-1}$ which is around 10 times larger than data by the end of Run 3. Tackling this data deluge at the HL-LHC requires significant upgrades in ATLAS and CMS detectors. In this proceedings, with the upgraded detectors, we discuss some prospects on the SM and Higgs physics at the HL-LHC.
\end{abstract}

An Alpine LHC Physics Summit (ALPS2018)

15-20 April, 2018

Obergurgl, Austria

${ }^{*}$ Speaker. 


\section{Introduction}

After the Higgs discovery, more data was required not only to check the consistency with the Standard Model (SM) but also to search for possible new physics. At the High Luminosity LHC (HL-LHC), the LHC will reach a peak instantaneous luminosity of $7.5 \times 10^{34} \mathrm{~cm}^{-2} \mathrm{~s}^{-1}$ in the middle of 2026. This upgrade will lead us to data corresponding an integrated luminosity of 3000 $\mathrm{fb}^{-1}$ which is around 10 times larger than data by the end of Run 3. With this large dataset, high precision less than $10 \%$ for measurements of Higgs couplings to other SM particles will be within reach and the Higgs self coupling can be explored through double Higgs production. New particles could also be revealed in loops or non-fundamental nature of the SM particles. However, tackling this data deluge at the HL-LHC is challenging and requires significant upgrades in ATLAS and CMS detectors.

In this proceedings, with the upgraded detectors, we discuss some prospects on the SM and Higgs physics at the HL-LHC. For these prospects, we adapted to mainly two approaches. One is to extrapolate from Run-1/2 results using the same analysis strategy. In this approach, it is required to correct the scale for higher center of mass energy $\sqrt{s}=14 \mathrm{TeV}$ and the yield of signal and background to $3000 \mathrm{fb}^{-1}$ and also for different object performance between Run-2 and HLLHC. The second approach is to emulate the expected performance through parameterization using the fast simulation. For systematic uncertainties, we applied two scenarios. In scenario 1 (S1), theoretical and experimental uncertainties remain the same as Run-1/2. In scenario 2 (S2), the theoretical uncertainty is reduced by half and the experimental uncertainty is scaled down by $1 / \sqrt{L}$. However, we should keep in mind that the systematic uncertainty will evolve with luminosity and time.

\section{SM}

\subsection{Vector boson scattering}

In the process of $\mathrm{pp} \rightarrow \mathrm{VVjj}$ where the vector bosons are radiated off from incoming quarks and scatter off each other, strong cancellation in the SM from double triple gauge (TGC), quartic gauge coupling (QGC) and Higgs boson exchange occur. Any beyond the SM addition to the scattering process would alter the cancellation of the SM. This results in cross section changes at high scattering center of mass energy [1]. In the study from the ATLAS experiment with the extended tracker up to $|\eta|<4.0$ and forward muon tagger, the signal yield increases by $14 \%$ while only increasing the background by $7.3 \%$ [2]. The significance and precision of $\mathrm{WWjj}$ cross section measurement can be improved by $15 \%$ and 13\%, respectively [2]. Figures 1 show the pseudorapidity distribution of the leading jets (left) covering high $\eta$ area and the dijet azimuthal separation $(|\Delta \phi(j, j)|)$ distribution (right).

\subsection{Double parton scattering}

With high luminosity, simultaneous scattering of multiple partons within proton can occur. The cross section of a double parton scattering process can be factorized into the cross sections for the two single partonic processes and a factor $\sigma_{\text {eff }}$ as $\sigma=\sigma_{1} \sigma_{2} / \sigma_{\text {eff }}$. The $\sigma_{\text {eff }}$ is an effective cross section determined by the transverse profile of partons and their overlaps in a collision. 

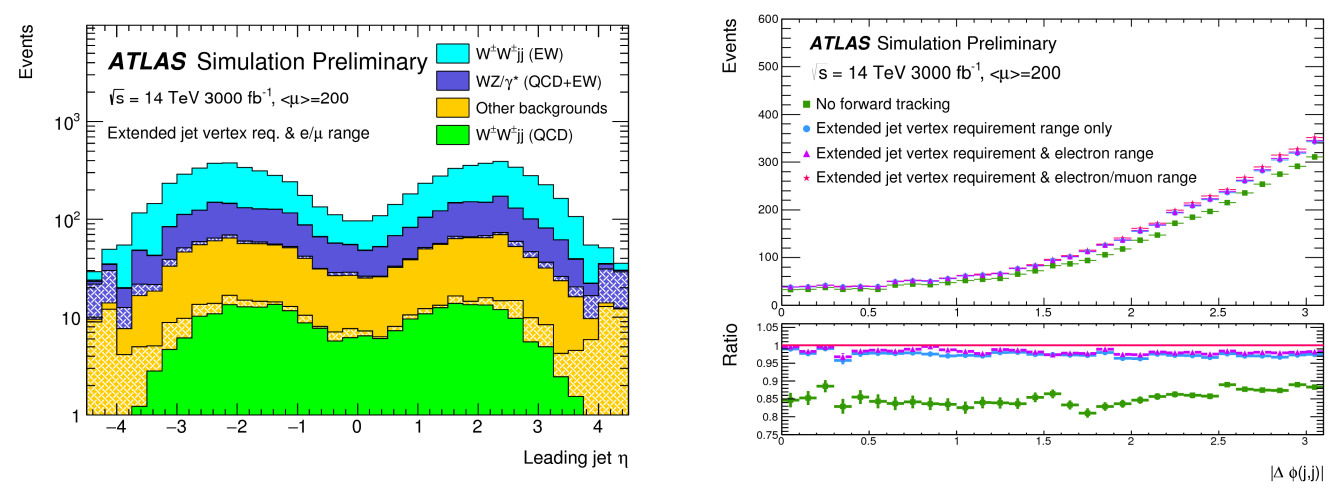

Figure 1: Pseudorapidity distribution of the leading jets (left) [2]. The dijet azimuthal separation $(|\Delta \phi(j, j)|)$ distribution for all channels combined (right). The four scenarios varying the jet vertex requirement and lepton $\eta$ ranges are shown [2].

Extrapolation is done based on the result from Run-2 in CMS with the decay products of muons from the same-sign $W$ bosons. In the case of the hypothesis of nonfactorization, the final state kinematics of the $W$ decay products can affect each other. The $\sigma_{e f f}$ can have a dependence on the pseudorapidities of the two muons, $\eta_{1} \cdot \eta_{2}$. Measuring the dependence as a function of $\eta_{1} \cdot \eta_{2}$ becomes possible with a large dataset at the HL-LHC. Strong sensitivity at high values for $\eta_{1} \cdot \eta_{2}$ with the large data set from the HL-LHC can be obtained [3]. From this differential measurement, we will learn something about the proton structure.

\section{$2.3 A_{F B}$ of $Z$ events}

Dilepton pairs are produced through the annihilation of a quark and antiquark through the $Z$ boson or a virtual photon. Forward-backward asymmetry of Drell-Yan events $\left(A_{F B}\right)$ is defined based on the angle $\left(\theta^{*}\right)$ of the lepton $\left(l^{-}\right)$in the Collins-Soper frame of the dilepton system:

$$
A_{F B}=\frac{\sigma_{F}-\sigma_{B}}{\sigma_{F}+\sigma_{B}}
$$

where $\sigma_{F}$ and $\sigma_{B}$ are the cross sections in the forward $\left(\cos \theta^{*}>0\right)$ and backward $\left(\cos \theta^{*}<0\right)$ hemispheres, respectively. Weak mixing angle is sensitive to forward-backward asymmetry of Drell-Yan events. In the HL-LHC, a study in ATLAS recently shows that forward tracker extension can boost the sensitivity by reconstructing forward electrons with better performance. Figures 2 show that the central-forward category is the one that carries the most sensitivity (left) and uncertainty is dominated by PDF now (right) [4]. Corresponding CMS result can be found in Ref. [5].

\subsection{FCNC}

Flavor changing neutral current (FCNC) is suppressed by the GIM mechanism in the SM. In the SM, the branching ratio of the decay of $t \rightarrow X q(X=H, \gamma, Z, g)$ is around $10^{-17}-10^{-12}$. A wide variety of models shows a strong dependence in the measurable FCNC quantities. Search is usually done in single top quark production and top quark decay from a top-quark pair production.

In the CMS experiment, searches for the coupling of $t \gamma q$ are done in a single top-quark production and a top-quark decay from a top-quark pair production. The search is pursued requiring highenergetic photon with $p_{\mathrm{T}}>50 \mathrm{GeV}$ and $|\eta|<2.5$. The signal distribution is clearly distinguished 

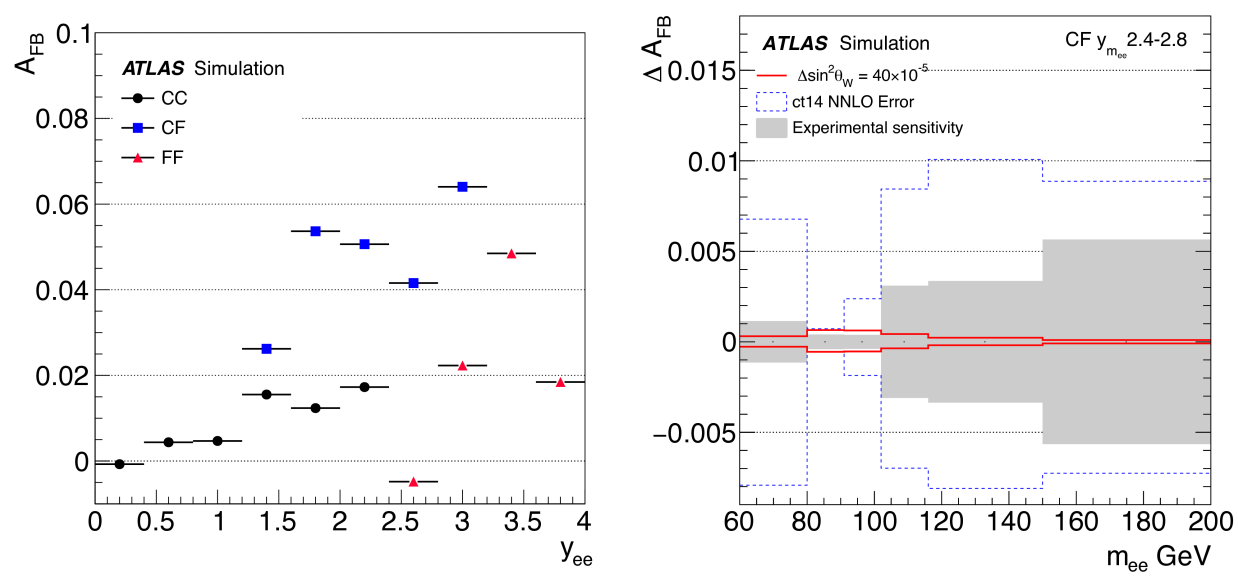

Figure 2: $\mathrm{A}_{F B}$ distribution as a function of the di-electron rapidity in the fiducial volume for central-central (CC), central-forward (CF) and forward-forward (FF) (left) [4]. Distribution of $\triangle A_{F B}$ as a function of mass for the CF class (right) [4].

in the $p_{\mathrm{T}}$ and $\eta$ distributions as shown in Figs. 3 (upper left and right). Fake photon background contribution is extrapolated from the results at $8 \mathrm{TeV}$. Figure 3 (lower right) shows the projections at the HL-LHC for comparing with current best limit for $\mathrm{B}(t \rightarrow q+Z)$ and $\mathrm{B}(t \rightarrow q+\gamma)$. The upper limits at 95\% CL on the branching fractions are $\mathrm{B}(t \rightarrow u+\gamma)<0.0027 \%$ and $\mathrm{B}(t \rightarrow c+\gamma)$ $<0.020 \%$.

In the ATLAS experiment, for the coupling of $t Z q$, events of the $t \rightarrow Z q$ are reconstructed using kinematic reconstruction minimizing $\chi^{2}$ value which is based on the constraints from the mass of $Z$ boson, $W$ boson and two top quarks from the $t \rightarrow W b$ decay and the $t \rightarrow Z q$ decay. Figure 3 (lower left) shows the $\chi^{2}$ distribution.

We can even close the window of FCNC parameter space for beyond the SM at the HL-LHC with dedicated studies.

\section{Higgs}

\subsection{Signal Strength}

From Run-2 with an integrated luminosity of $35.9 \mathrm{fb}^{-1}$, the signal strength relative to the standard model is close to unity. The Higgs signal strength at the HL-LHC is extrapolated based on the results with an integrated luminosity of $12.9 \mathrm{fb}^{-1}$ at $13 \mathrm{TeV}$. Figure 4 (left) shows projected $68 \%$ uncertainties for the Higgs boson signal strength relative to the standard model from various channels in the ATLAS. Figure 4 (right) shows the $H \rightarrow Z Z$ signal strength relative to the standard model $\left(\mu^{\mathrm{ZZ}}\right)$, inclusively and per production mode in the CMS with the integrated luminosity of $3000 \mathrm{fb}^{-1}$.

In ATLAS, with $3000 \mathrm{fb}^{-1}$, a few-percent level can be reached in the diphoton and diboson final states. In these final states, $9 \%$ precision including theory uncertainty can be obtained using the same analysis strategy from Run-1 and experimental uncertainties of around 5\% is reachable. For further improvement, theoretical uncertainties from QCD scale, PDF and $\alpha_{S}$ need to be reduced. 

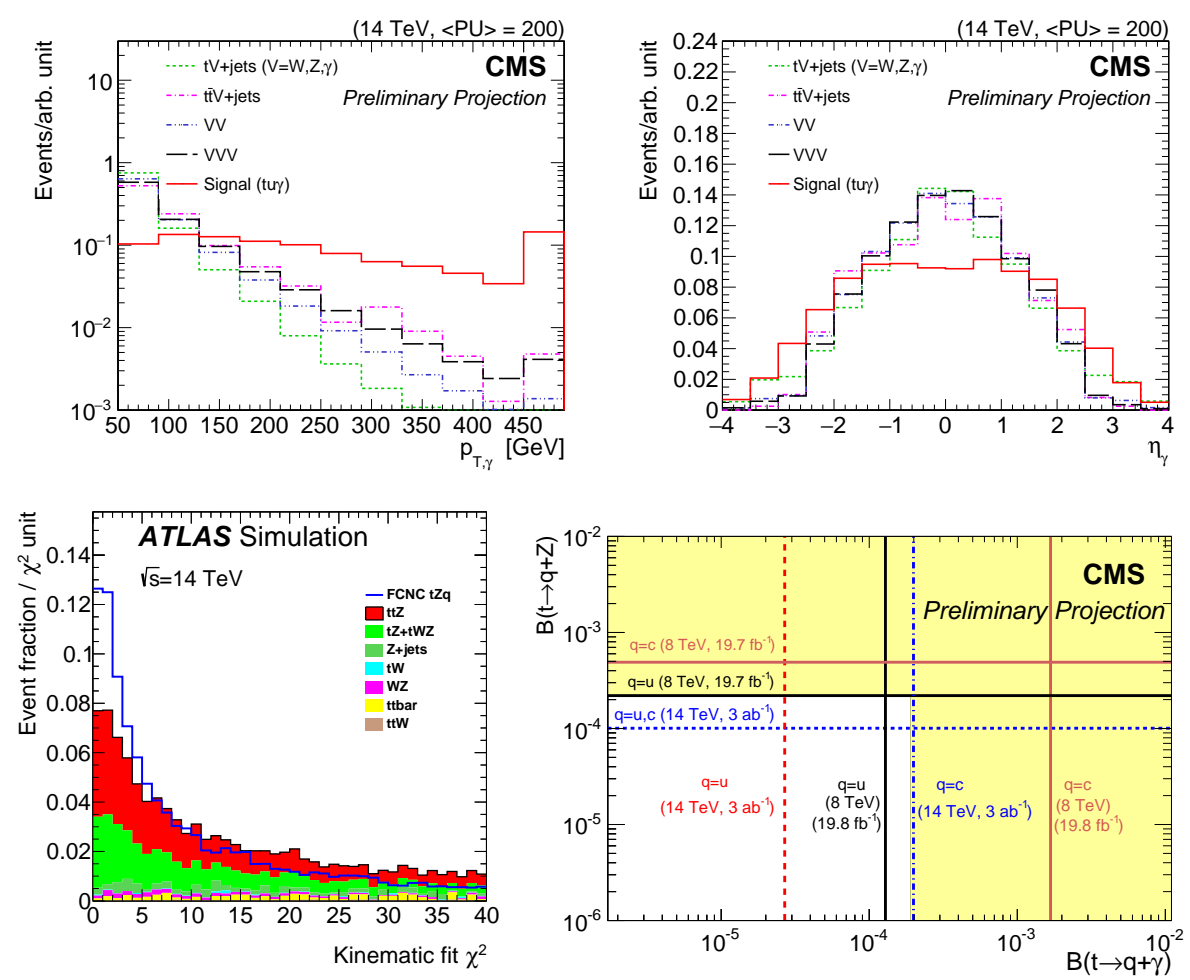

Figure 3: Transverse momentum (upper left) and pseudorapidity (uppwer right) of the photon candidates from $t+\gamma$ production due to $t \gamma q$ FCNC interaction and various background processes [8]. Distribution of $\chi^{2}$ after kinematic fit [6] (lower left). Expected upper limits at 95\% confidence level on $\mathrm{B}(t \rightarrow q+Z)$ and $\mathrm{B}(t \rightarrow q+\gamma)$ are shown [8] (lower right). The horizontal dashed line indicates upper limit on $\mathrm{B}(t \rightarrow q+Z)$ and the two vertical dashed and dashed-dotted lines indicate the projections on $\mathrm{B}(t \rightarrow q+\gamma)$ at the HL-LHC.

Statistical uncertainty is expected to be better by a factor of around 9 compared to the uncertainty from current results. For other decay modes such as $\mathrm{H} \rightarrow \mathrm{b} \bar{b}$ and $\mathrm{H} \rightarrow \tau \tau$, we expect the precision about 10-20\% level where signal modeling including QCD scale is dominant uncertainty.

In CMS, a few-percent level precision dominated by systematic uncertainty can also be reached with an integrated luminosity of $3000 \mathrm{fb}^{-1}$ for inclusive measurements for the signal strength. For $V B F, V H$ and $t \bar{t} H$ productions, it is still statistically limited (see Fig. 4).

\subsection{Higgs cross section}

Precision timing measurements for photons and charged particles would improve pileup suppression in vertex reconstruction, isolation, jets and MET physics objects. This improvement is clearly visible in the mass resolution and uncertainty. Approximately $15 \%$ improvement in the statistical uncertainty is expected from the pessimistic to the optimistic scenario.

Through the differential cross section measurements, we can even improve the sensitivity to physics beyond the SM. This can also probe perturbative QCD calculation. In Run-2 scenario, in ATLAS, the uncertainty is between $8-17 \%$. In optimistic scenario where the experimental uncertainties are reduced by a factor of 2 , the uncertainty is between $6-14 \%$. This improves the current result by more than factor of 6 . 

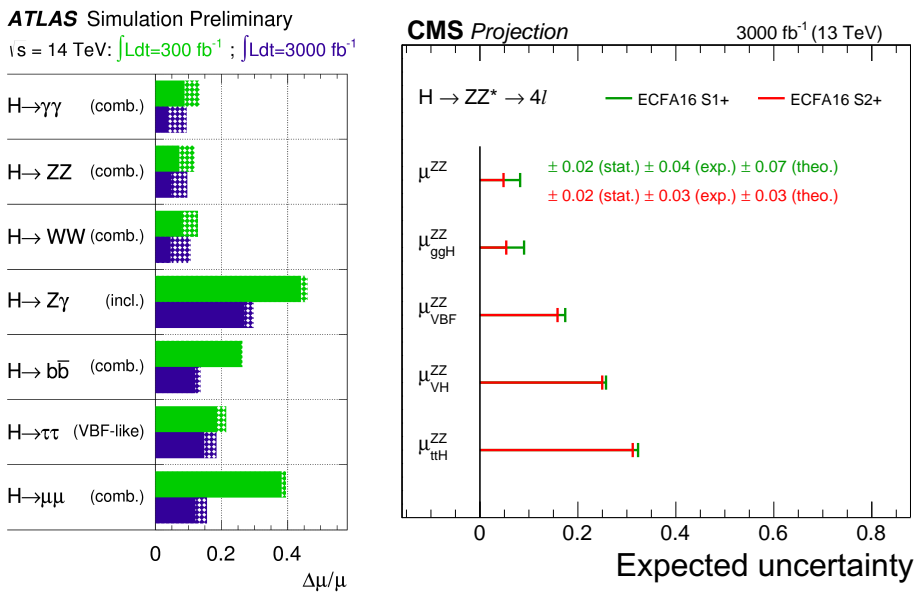

Figure 4: Relative uncertainty on the signal strength $\mu$ for all Higgs final states. The hased areas indicate the increase of the estimated error due to current theory systematic uncertainties (left) [7]. The projected 68\% CL uncertainty in the Higgs boson signal strength (right) [9].

In the CMS experiment, projection is done for the fiducial differential cross section of $p_{\mathrm{T}}$ of the Higgs $\left(\mathrm{H} \rightarrow \mathrm{ZZ}^{*} \rightarrow 4 l\right)$. Figure 5 (right) shows the $p_{\mathrm{T}}$ of 4 muons. Probing the high $p_{\mathrm{T}}$ phase space sensitive to possible deviation from the SM. Statistical uncertainty ranges from 4 to $9 \%$. At high $p_{\mathrm{T}}>200 \mathrm{GeV}$, statistical uncertainty becomes dominant.

\subsection{Rare process}

The measurement of the couplings of the Higgs boson to fermions is one of the important studies to check the consistency with the SM and to search for possible deviations. At the HLLHC, the $\mathrm{H} \rightarrow \mu \mu$ process has been used as a benchmark for detector upgrade. This signature can serve as a probe for coupling to fermion of second generation. The signature of the $\mathrm{H} \rightarrow$ $\mu \mu$ process has a small branching fraction of $2.2 \times 10^{-4}$ in the SM. With the large datasets of the HL-LHC, searching for rare decays of the Higgs boson becomes accessible.

The sensitivity at the HL-LHC for this channel was studied from the ATLAS experiment. The projection is based on a selection and event classification similar to that used for Run-2 data [10]. In the ATLAS experiment, the resolution on the mass is expected to be better by $25 \%$ compared to the one with Run-2 layout thanks to the superior performance of the Inner Tracker.

In the CMS experiment, for cross section and coupling, the upgraded detector achieves about $65 \%$ better invariant mass resolution with the reduced material budget and improved spatial resolution of the upgraded tracker. This leads to an uncertainty of 5\% for couplings to muons and $10 \%$ for the cross section measurement with $3000 \mathrm{fb}^{-1}$ [11]. Figure 5 shows the dimuon invariant mass distribution of $\mathrm{H} \rightarrow \mu \mu$ decays for muons in the central region at the HL-HLC.

\subsection{Double Higgs production}

Higgs self coupling is the key parameter to understand the nature of the Higgs boson in the SM. The coupling can be measured through the di-Higgs production. However, it interferes destructively with the di-Higgs production through a box diagram. The di-Higgs production through 

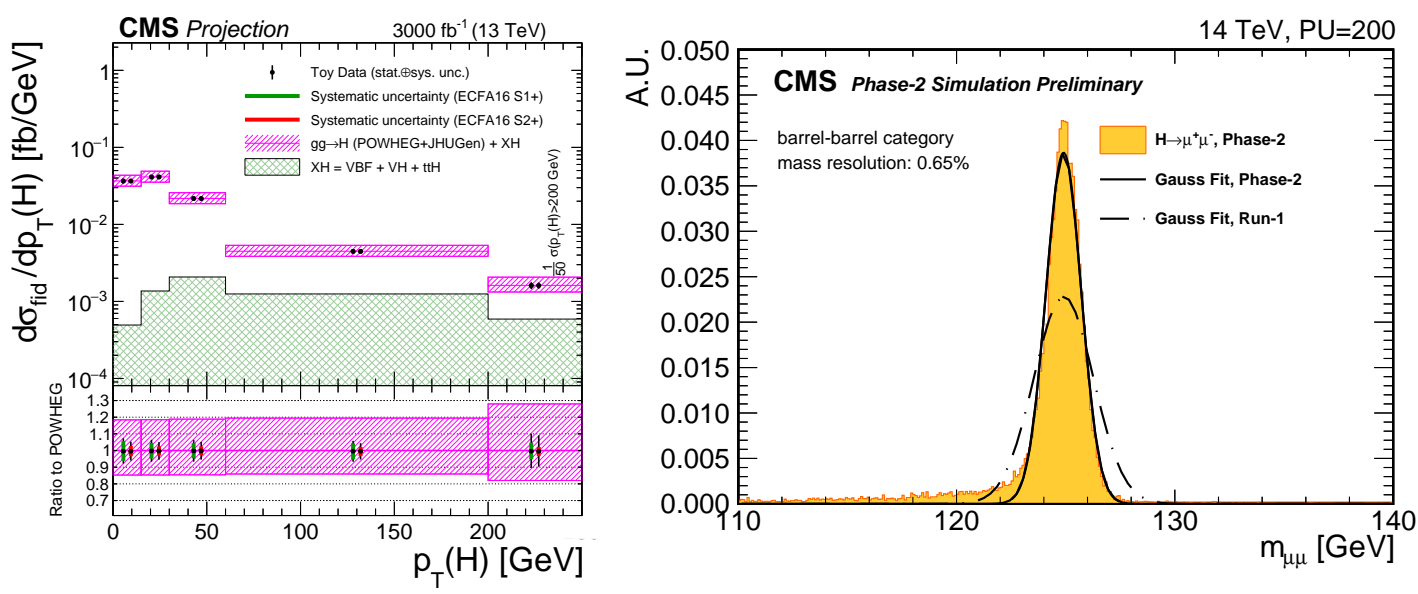

Figure 5: Projection for the differential fiducial cross section measurement of the Higgs boson transverse momentum (left) [9]. The di-muon invariant mass distribution for $\mathrm{H} \rightarrow \mu^{+} \mu^{-}$decays for muons in the central region (right) [11].

gluon-gluon fusion in the SM is around $40 \mathrm{fb}^{-1}$ at a center of mass $14 \mathrm{TeV}$. With an integrated luminosity of $3000 \mathrm{fb}^{-1}, 120 \mathrm{~K}$ di-Higgs events are expected.

The largest branching ratio comes from the final states of $b \bar{b} b \bar{b}$ from the $\mathrm{H} \rightarrow b \bar{b}$. However, it is very challenging to pursue these searches due to large backgrounds mostly from the $t \bar{t}$. In the ATLAS experiment, in Run-1/2, the most sensitivity channel to Higgs boson pair production came from the $H H \rightarrow b \bar{b} b \bar{b}$. With Run-2 data, a signal strength of $\mu=\sigma / \sigma_{S M} \geq 29$ was excluded at $95 \%$ confidence level. The projections at the HL-LHC are based on the results from the Run-2 data in the assumption of the upgraded detector and improvements to reconstruction algorithms. At the HLLHC, the upper limit on the cross section relative to the standard model prediction is $\mu=\sigma / \sigma_{S M}$ $=1.5$ without taking into account systematic uncertainties [13]. Higgs boson self coupling should be within the range between $0.2<\lambda_{H H H} / \lambda_{S M}<7.0$ without systematic uncertainties and $-3.5<$ $\lambda_{H H H} / \lambda_{S M}<11$ with taking into account the systematic uncertainties from the results in 2016. However, it is very challenging to trigger the signal events with the final state of $b \bar{b} b \bar{b}$ as it is sensitive to trigger threshold on $p_{\mathrm{T}}$ with 4 jet requirement. In the CMS experiment, the expected upper limit on the signal strength is 2.9 assuming that the uncertainties in other backgrounds are unchanged with respect to the results from Run-2. This leads to an expected significance is 0.67 [9].

The final state of $\gamma \gamma b \bar{b}$ is one of the most promising channels for the Higgs self-coupling. An advantage of this channel is to have a narrow mass peak from the Higgs boson decaying $\gamma \gamma$ even though its branching ratio is small around $0.3 \%$ which is corresponding to around 300 events with an integrated luminosity of $3000 \mathrm{fb}^{-1}$. In this final state, the $\gamma+$ jets would be the main background when one of jets is misidentified as a photon. In the ATLAS experiment, the expected number of final events is around 10 events for the signal and 91 events for the background. The invariant mass distribution of diphoton pairs is shown in Fig. 6 (left). Splitting the events into two categories depending on the direction of the photons (barrel-barrel and other) to improve the sensitivity, an expected significance is $1.05 \sigma$ [12]. Higgs boson self-coupling should be in - $0.8<\lambda_{H H H} / \lambda_{S M}<$ 7.7 not including systematic uncertainty. The cross-section limit indicated as dashed line together 
with the $\pm 1 \sigma$ and $\pm 2 \sigma$ uncertainty bands is shown in Fig. 6 (right). In the CMS experiment, the most sensitive channel comes from the decay mode of $H H \rightarrow \gamma \gamma b \bar{b}$ with a significance of 1.47 standard deviations when only considering statistical uncertainty [9]. The scenario assumes the btagging uncertainty of $1 \%$ level and upgraded detector performance with an average of 200 pileup events.
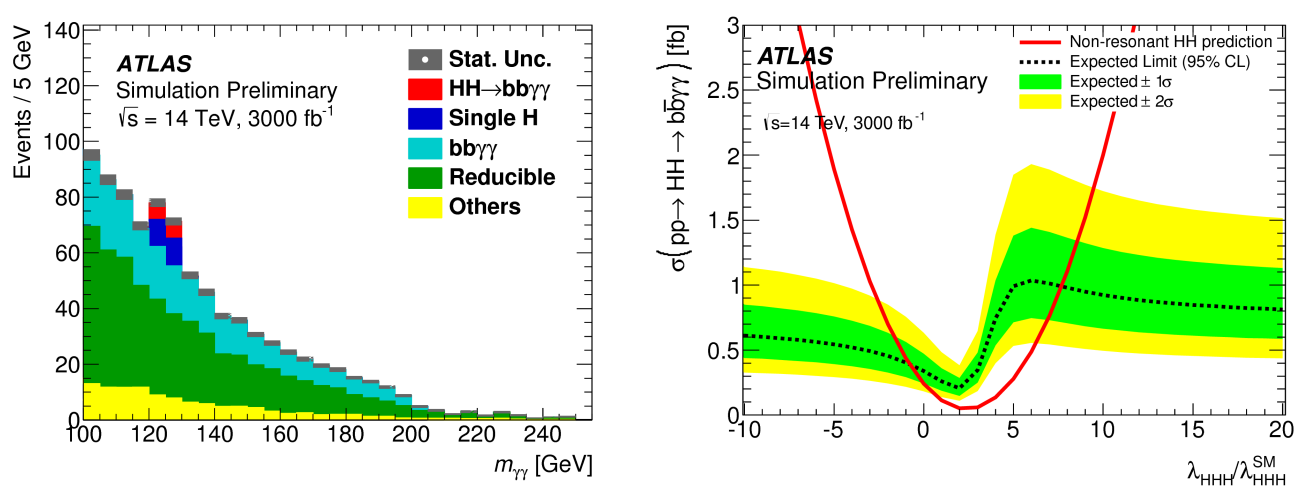

Figure 6: Diphoton invariant mass distribution (left) [12] and expected 95\% confidence level upper limit on the cross-section $\sigma(\mathrm{HH} \rightarrow b \bar{b} \gamma \gamma)$ with $3000 \mathrm{fb}^{-1}$ of data at the ATLAS experiment (right). The systematic uncertainties are not taken into account [12].

As shown in Fig. 7 (left), each of the channel alone is insufficient to observe the Higgs boson pair production. However, combining with these channels including other decay modes such as $b \bar{t} \tau \bar{\tau}$ is promising. The expected significance in the $b \bar{b} \tau \bar{\tau}$ final state is shown in Fig. 7 (right).
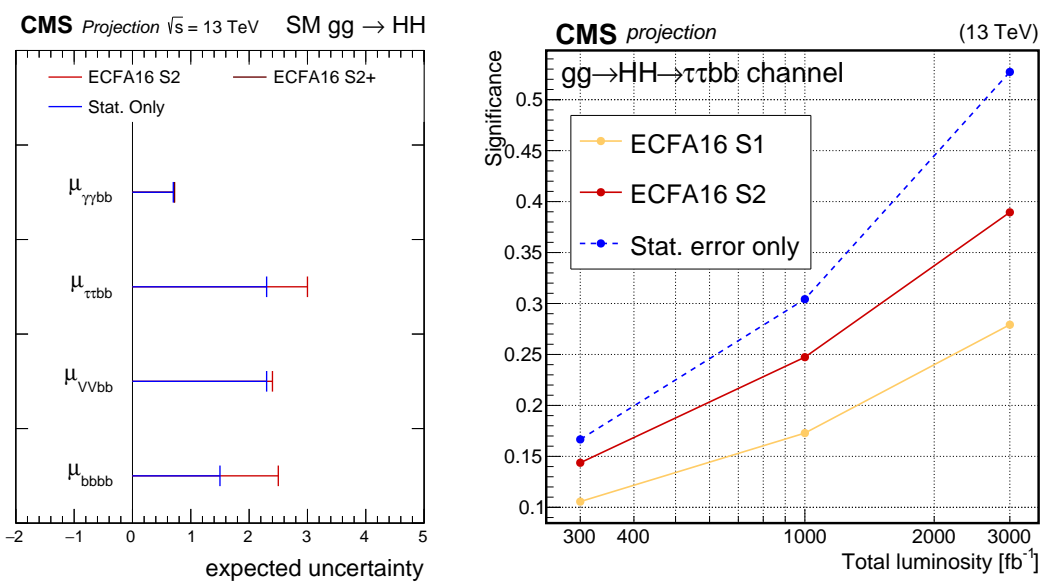

Figure 7: Projection of the sensitivity to the di-Higgs production in various final states at $3000 \mathrm{fb}^{-1}$ (left). Projection of the sensitivity to the SM $H H \rightarrow \tau \tau b \bar{b}$ production as a function of the luminosity based on the $13 \mathrm{TeV}$ preliminary results under the different scenarios (right) [9].

\section{Conclusion}

In the middle of 2026, LHC will reach a peak instantaneous luminosity of $7.5 \times 10^{34} \mathrm{~cm}^{-2} \mathrm{~s}^{-1}$. This upgrade will lead us to data corresponding an integrated luminosity of $3000 \mathrm{fb}^{-1}$ which is 
more than 10 times larger than data by the end of Run 3. The large dataset will allow us to perform precision measurements with excellent upgraded detector performance including better reconstruction techniques and pileup mitigation. With the large dataset, we can also explore corners of phase space which are sensitive to possible new physics. This is just beginning of the studies at the HLLHC. There is a coordinated effort by ATLAS, CMS and theorists to update and combine results which will be documented in a CERN Yellow Report at the end of the year. Better precisions should come from the optimized Phase- 2 detectors documented in TDRs and from the better analysis tools developed for Run-2.

\section{References}

[1] CMS Collaboration, CMS-PAS-SMP-14-008, https://cds.cern.ch/record/2220831.

[2] ATLAS Collaboration, ATL-PHYS-PUB-2017-023, https://cds.cern.ch/record/2298958.

[3] CMS Collaboration, CMS-TDR-016, https://cds.cern.ch/record/2283189.

[4] ATLAS Collaboration, https://atlas.web.cern.ch/Atlas/GROUPS/PHYSICS/PLOTS/ITK-2018-001.

[5] CMS Collaboration, CMS-PAS-FTR-17-001, http://cds.cern.ch/record/2294888.

[6] ATLAS Collaboration, ATL-PHYS-PUB-2016-019, https://cds.cern.ch/record/2209126.

[7] ATLAS Collaboration, ATL-PHYS-PUB-2014-016, https://cds.cern.ch/record/1956710.

[8] CMS Collaboration, CMS-PAS-FTR-16-006, cds.cern.ch/record/2262606.

[9] CMS Collaboration, CMS-PAS-FTR-16-002, http://cds.cern.ch/record/2266165.

[10] ATLAS Collaboration, ATLAS-TDR-026, http://cds.cern.ch/record/2285580.

[11] CMS Collaboration, CMS-TDR-17-001, https://cds.cern.ch/record/2272264.

[12] ATLAS Collaboration, ATL-PHYS-PUB-2017-001, https://cds.cern.ch/record/2243387.

[13] ATLAS Collaboration, ATL-PHYS-PUB-2016-024, https://cds.cern.ch/record/2221658. 